Old Dominion University

ODU Digital Commons

1995

\title{
Exclusive Electron Scattering from Deuterium at High Momentum Transfer
}

H. J. Bulten

P. L. Anthony

R. G. Arnold

J. Arrington

E. J. Beise

See next page for additional authors

Follow this and additional works at: https://digitalcommons.odu.edu/physics_fac_pubs

Part of the Astrophysics and Astronomy Commons, Elementary Particles and Fields and String Theory Commons, and the Quantum Physics Commons

\section{Original Publication Citation}

Bulten, H.J., Anthony, P.L., Arnold, R.G., ... Kuhn, S.E., White, J.L., Zeidman, B.(1995). Exclusive electron scattering from deuterium at high momentum transfer. Physical Review Letters, 74(24), 4775-4778. https://doi.org/10.1103/PhysRevLett.74.4775

This Article is brought to you for free and open access by the Physics at ODU Digital Commons. It has been accepted for inclusion in Physics Faculty Publications by an authorized administrator of ODU Digital Commons. For more information, please contact digitalcommons@odu.edu. 


\section{Authors}

H. J. Bulten, P. L. Anthony, R. G. Arnold, J. Arrington, E. J. Beise, E. Belz, K. van Bibber, P. E. Bosted, J.F.J. van den Brand, M. S. Chapman, K. P. Coulter, F. S. Dietrich, R. Ent, M. Epstein, B. W. Filippone, H. Gao, R.A. Gearhart, D. F. Geesaman, J.-O. Hansen, R. J. Holt, H. E. Jackson, C. E. Jones, C. E. Keppel, E. Kinney, S. E. Kuhn, K. Lee, W. Lorenzon, A. Lung, N.C.R. Makins, D. J. Margaziotis, R.D. McKeown, R. G. Milner, B.

Mueller, J. Napolitano, J. Nelson, T.G. O'Neill, V. Papavassiliou, G. G. Petratos, D. H. Potterveld, S. E. Rock, M. Spengos, Z. M. Szalata, L. H. Tao, J. L. White, and B. Zeidman 


\section{Exclusive Electron Scattering from Deuterium at High Momentum Transfer}

H. J. Bulten, ${ }^{1}$ P. L. Anthony, ${ }^{2}$ R. G. Arnold, ${ }^{3}$ J. Arrington, ${ }^{4}$ E. J. Beise,,${ }^{4, *}$ E. Belz,,${ }^{4,}{ }^{\dagger}$ K. van Bibber, ${ }^{2}$ P. E. Bosted, ${ }^{3}$ J. F. J. van den Brand, ${ }^{1}$ M. S. Chapman, ${ }^{5}$ K. P. Coulter, ${ }^{6,{ }^{\ddagger}}$ F. S. Dietrich, ${ }^{2}$ R. Ent,,${ }^{5,}$ M. Epstein, ${ }^{7}$ B. W. Filippone, ${ }^{4}$ H. Gao, ${ }^{4, \|}$ R. A. Gearhart, ${ }^{8}$ D. F. Geesaman, ${ }^{6}$ J.-O. Hansen, ${ }^{5}$ R. J. Holt, ${ }^{6, \|}$ H. E. Jackson, ${ }^{6}$ C. E. Jones, ${ }^{1, \pi}$ C. E. Keppel, ${ }^{3,}{ }^{* *}$ E. Kinney, ${ }^{9}$ S. E. Kuhn, ${ }^{10,+\dagger}$ K. Lee, ${ }^{5}$ W. Lorenzon, ${ }^{4, \neq \ddagger}$ A. Lung, ${ }^{3,8 \S}$ N. C. R. Makins, ${ }^{5, l l}$ D. J. Margaziotis, ${ }^{7}$ R. D. McKeown, ${ }^{4}$ R. G. Milner, ${ }^{5}$ B. Mueller, ${ }^{4}$ J. Napolitano, ${ }^{11}$ J. Nelson, ${ }^{5, \| l}$ T. G. O'Neill, ${ }^{4, \pi}$ V. Papavassiliou, ${ }^{6}$ G. G. Petratos, ${ }^{8, \text { ITI }}$ D. H. Potterveld, ${ }^{6}$ S. E. Rock, ${ }^{3}$ M. Spengos, ${ }^{3}$ Z. M. Szalata, ${ }^{3}$ L. H. Tao, ${ }^{3}$ J. L. White, ${ }^{3}$ and B. Zeidman ${ }^{6}$

${ }^{1}$ University of Wisconsin, Madison, Wisconsin 53706

${ }^{2}$ Lawrence Livermore National Laboratory, Livermore, California 94550

${ }^{3}$ The American University, Washington, D.C. 20016

${ }^{4}$ W. K. Kellogg Radiation Laboratory, California Institute of Technology, Pasadena, California 91125

${ }^{5}$ Laboratory for Nuclear Science, Massachusetts Institute of Technology, Cambridge, Massachusetts 02139

${ }^{6}$ Argonne National Laboratory, Argonne, Illinois 60439

${ }^{7}$ California State University, Los Angeles, California 90032

${ }^{8}$ Stanford Linear Accelerator Center, Stanford, California 94309

${ }^{9}$ University of Colorado, Boulder, Colorado 80309

${ }^{10}$ Stanford University, Stanford, California 94305

${ }^{11}$ Rensselaer Polytechnic Institute, Troy, New York 12180

(Received 4 November 1994)

Cross sections are presented for the reaction ${ }^{2} \mathrm{H}\left(e, e^{\prime} p\right) n$ for momentum transfers in the range $1.2 \leq Q^{2} \leq 6.8(\mathrm{GeV} / c)^{2}$ and for missing momenta from 0 to $250 \mathrm{MeV} / c$. The longitudinal-transverse interference structure function has been separated at $Q^{2}=1.2(\mathrm{GeV} / c)^{2}$. The observables are compared to calculations performed in nonrelativistic and relativistic frameworks. The data are best described by a fully relativistic calculation.

PACS numbers: $25.30 . \mathrm{Fj}, 25.10 .+\mathrm{s}$

Quasielastic electron-scattering experiments can be used to explore single-particle properties of the nucleus. Until recently, such experiments were performed at relatively moderate four-momentum transfer squared $\left(Q^{2}\right)$, typically $Q^{2} \approx 0.2(\mathrm{GeV} / c)^{2}$. With the advent of a new generation of electron-scattering facilities (e.g., CEBAF) capable of delivering higher beam energies, experiments are planned at higher momentum transfer. These experiments may be sensitive to new phenomena, for example, the QCD prediction of color transparency [1] or manifestations of quark-exchange effects [2]. In order to interpret such new data at high $Q^{2}$ correctly, the $\left(e, e^{\prime} p\right)$ cross section must be precisely understood in this kinematic regime.

The analysis of quasielastic $\left(e, e^{\prime} p\right)$ experiments has generally been performed in a nonrelativistic framework, to facilitate the calculation of nuclear wave functions in the initial and final states. For the current operator often a nonrelativistic reduction is used, truncated at lowest order in $p / M$, where $p(M)$ is the proton momentum (mass). At high $Q^{2}$ this approximation is invalid since the proton momentum is not small compared to its mass.

Apart from relativistic ingredients in the $\left(e, e^{\prime} p\right)$ reaction mechanism, new phenomena may occur due to the fact that the wavelength of the photon becomes small $\left[1 / Q \approx 0.08 \mathrm{fm}\right.$ at $\left.Q^{2}=6.8(\mathrm{GeV} / c)^{2}\right]$ with respect to the size of the nucleon $\left(\langle r\rangle_{\mathrm{rms}} \approx 0.8 \mathrm{fm}\right)$. It is thus not clear a priori that quasielastic scattering still can be described in terms of the impulse approximation using nucleon-meson fields.

It is well known that in the one-photon exchange approximation the spin-averaged sixfold $\left(e, e^{\prime} p\right)$ coincidence cross section can be written as [3]

$$
\frac{d^{6} \sigma}{d \Omega_{e} d \Omega_{p} d E d E_{p}}=K \sigma_{\mathrm{Mott}} \frac{Q^{2}}{\vec{q}^{2}}\left\{W_{L}+\frac{1}{\varepsilon} W_{T}-\sqrt{\frac{\varepsilon+1}{\varepsilon}} \cos (\phi) W_{L T}+\cos (2 \phi) W_{T T}\right\}
$$

with $\varepsilon\left(=\left[1+2 \mathbf{q}^{2} / Q^{2} \tan ^{2}\left(\theta_{e} / 2\right)\right]^{-1}\right)$ the virtual-photon polarization. $E\left(E_{p}\right)$ is the electron (proton) energy, $\Omega_{e}$ $\left(\Omega_{p}\right)$ is the electron (proton) solid angle, $\mathbf{q}$ is the threemomentum transfer, $\theta_{e}$ is the electron-scattering angle, $K$ is a kinematical factor, and $\phi$ is the angle between the electron-scattering plane and the reaction plane, defined by the direction of the momentum transfer and the knocked-out proton.

For scattering from a stationary free nucleon, expression (1) reduces to the Rosenbluth formula, which depends solely on the longitudinal and transverse structure functions $W_{L}$ and $W_{T}$. The structure function $W_{L T}\left(W_{T T}\right)$ 
arises from the interference of the charge (transverse current) term with the transverse current term of the nuclear current operator, where transverse refers to the components perpendicular to the virtual-photon momentum. They are zero if the momentum of the knocked-out proton is parallel to the momentum transfer.

From Eq. (1) it is clear that the value of the longitudinal-transverse interference structure function, $W_{L T}$, can be determined by performing measurements of the cross section at $\phi=0$ and $\phi=\pi$, keeping all other kinematic quantities constant. To reduce systematic uncertainties the data are represented in terms of the asymmetry $A_{\phi}$ :

$$
\begin{gathered}
A_{\phi}=\frac{\sigma(\phi=0)-\sigma(\phi=\pi)}{\sigma(\phi=0)+\sigma(\phi=\pi)} \\
\left(=\frac{\sqrt{(\varepsilon+1) \varepsilon} W_{L T}}{\varepsilon W_{L}+W_{T}+\varepsilon W_{T T}}\right) .
\end{gathered}
$$

In general a dynamical model is needed to calculate the nuclear structure functions $W_{i}$. In the plane-wave impulse approximation (PWIA) the cross section factorizes [3] into a term containing the electron-proton cross section and a term depending on the nuclear wave function. The latter term is common to all structure functions, hence in PWIA the asymmetry $A_{\phi}$ exclusively depends on the nucleon current operator.

It has been claimed [4] that already at low $Q^{2}[\approx$ $\left.0.2(\mathrm{GeV} / c)^{2}\right]$ relativistic effects may become important in $A_{\phi}$ and consequently $W_{L T}$, whereas for $W_{L}$ and $W_{T}$ [5] the nonrelativistic and relativistic calculations gave about the same results.

In the present experiment the $\left(e, e^{\prime} p\right)$ cross section is measured for quasielastic scattering from the deuteron for $Q^{2}$ values between 1.2 and $6.8(\mathrm{GeV} / c)^{2}$ and $W_{L T}$ for $Q^{2}=1.2(\mathrm{GeV} / c)^{2}$. Exact calculations for realistic nucleon-nucleon $(N N)$ potentials can be performed for the two-body system. This allows for an important benchmark study of the $\left(e, e^{\prime} p\right)$ quasielastic reaction mechanism using deuterium. The dependence of the cross section on $Q^{2}$ is used to investigate the validity of the impulse approximation for quasielastic scattering while $W_{L T}$ has been extracted in order to determine the importance of relativistic effects in the cross section.

The experiment has been performed using the spectrometers in end station A of the Stanford Linear Accelerator Center (SLAC) [6]. The electron beam was accelerated to energies between 2.0 and $5.1 \mathrm{GeV}$ and had a duty factor of $0.02 \%$. The scattered electrons and recoiling protons were detected in two magnetic spectrometers with a maximum momentum of 1.6 and $4.5 \mathrm{GeV} / c$, respectively.

A coincidence time resolution of $0.8 \mathrm{~ns}$ (FWHM) was achieved. Uncertainties (FWHM) are $0.1 \%$ for the beam energy, $0.5 \mathrm{mrad}$ for the angles of the scattered electron and the knocked-out proton, 9 (28) $\mathrm{MeV}$ in missing energy, and, on average, $10(45) \mathrm{MeV} / c$ in missing momentum $\left(p_{m}\right)$ at $Q^{2}=1.2(6.8)(\mathrm{GeV} / c)^{2}$. The centroid of the missing-momentum distribution could be determined to
TABLE I. Kinematics for the ${ }^{2} \mathrm{H}\left(e, e^{\prime} p\right) n$ measurements.

\begin{tabular}{cccccc}
\hline \hline $\begin{array}{c}Q^{2} \\
{\left[(\mathrm{GeV} / c)^{2}\right]}\end{array}$ & $\begin{array}{c}E_{e} \\
(\mathrm{GeV})\end{array}$ & $\begin{array}{c}E_{e}^{\prime} \\
(\mathrm{GeV})\end{array}$ & $\begin{array}{c}p^{\prime} \\
(\mathrm{GeV} / c)\end{array}$ & $\begin{array}{c}\theta_{e} \\
(\mathrm{deg})\end{array}$ & $\begin{array}{c}\theta_{p} \\
(\mathrm{deg})\end{array}$ \\
\hline 1.20 & 2.02 & 1.39 & 1.28 & 38.8 & $35.9-50.2$ \\
3.06 & 3.19 & 1.47 & 2.45 & 47.7 & 27.7 \\
5.00 & 4.21 & 1.47 & 3.54 & 53.4 & 19.5 \\
6.77 & 5.12 & 1.47 & 4.49 & 56.6 & 15.9 \\
\hline \hline
\end{tabular}

$\pm 1.3 \mathrm{MeV} / c$. Details on the experimental setup and the data analysis can be found elsewhere $[1,7]$.

The kinematics used in the experiment are given in Table I. The extraction of $W_{L T}$ from the coincidence cross section is sensitive to the treatment of radiative effects. Wasson et al. [8] extended the approach of Mo and Tsai [9] to describe the radiative effects in the $\left(e, e^{\prime} p\right)$ reaction. The soft photon approximation is used, and proton radiation is included. The procedure has been extensively tested using ${ }^{1} \mathrm{H}\left(e, e^{\prime} p\right)$ data [7].

Figure 1 shows the coincidence cross section, integrated between -15 and $25 \mathrm{MeV}$ in missing energy, as a function of missing momentum [10] for $Q^{2}=1.2,3.1$, 5.0 , and $6.8(\mathrm{GeV} / c)^{2}$. The systematic error of $8.2 \%$, which is mainly due to the uncertainty in the detection phase space $(5 \%)$, the proton angle $(4 \%)$, and the radiative corrections $(3 \%)$, is not included in the error bars. The

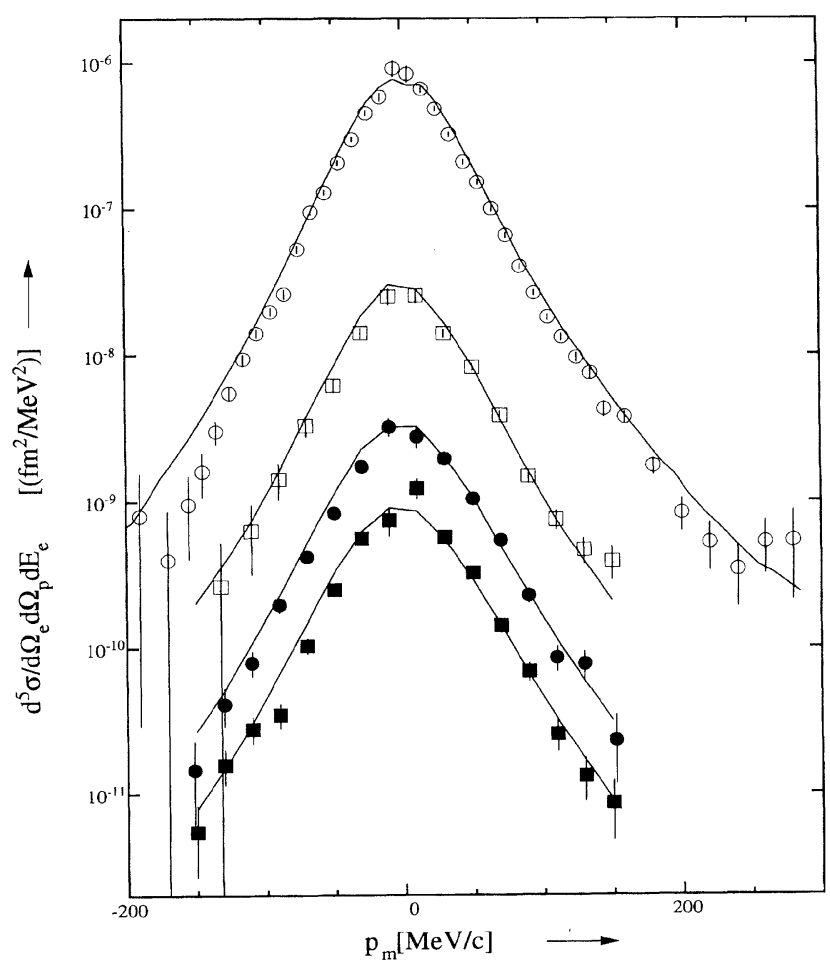

FIG. 1. Cross section for the reaction ${ }^{2} \mathrm{H}\left(e, e^{\prime} p\right) n$ as a function of the missing momentum. The open circles, open squares, filled circles, and filled squares represent the data at four-momentum transfer squared of 1.2, 3.0, 5.1, and $6.8(\mathrm{GeV} / c)^{2}$, respectively. 
curves represent the results of a PWIA calculation, based on the prescription $\sigma_{c c 1}$ of de Forest [11] for the off-shell electron-proton cross section and the Paris spectral function. The calculations incorporated the acceptance of the experimental setup using a Monte Carlo code which also takes radiative effects into account. The curves exceed the data on average by about $10 \%$. Note that a reduction of the cross section is to be expected due to the final-state interaction. Reasonable agreement is obtained for all $Q^{2}$, and the data are consistent with a description in terms of the quasifree scattering mechanism.

The reaction mechanism is studied in more detail by comparing the data to several modern calculations. In Tjon's relativistic field theoretical model [12] for the reaction ${ }^{2} \mathrm{H}\left(e, e^{\prime} p\right) n$, the Bethe-Salpeter equation is solved for a one-boson exchange potential. Relativistic and nonrelativistic contributions to the observables are separated by making a nonrelativistic reduction of the current operator through a second order Foldy-Wouthuysen [13] transformation and projecting out the positive-energy states of the proton wave function. The nonrelativistic calculations of Beck and Arenhövel [14] start from the Schrödinger equation. Their relativistic calculations include third and fourth order terms in $p / M_{p}$ in the current operator and contributions from the boost operator that translates the initial-state wave function from the laboratory frame to the center-of-mass frame. In addition, the cross section has been evaluated using the $\sigma_{c c 1}$ prescription [11] and the Paris two-body potential.

The coincidence cross section for the measurement at $Q^{2}=1.2(\mathrm{GeV} / c)^{2}$ is shown in Fig. 2. It is seen that the nonrelativistic calculation of Arenhövel significantly exceeds the data. The relativistic calculations of Arenhövel and Tjon and the prescription $K \sigma_{c c 1}$ give a good description of the cross section, except for $p_{m}<-125 \mathrm{MeV} / c$ $(\phi=0)$, where the data have limited statistical accuracy.

We have investigated the influence of the NN potential on the calculated cross sections using Arenhövel's treatment including final-state interaction (FSI), mesonexchange currents (MEC), and isobar configuration (IC) contributions. The results for $A_{\phi}$ using the Paris, Bonn, Nijmegen, and Argonne V14 NN potential differ by less than $2 \%$ for missing momenta below $150 \mathrm{MeV} / c$ and therefore do not influence our conclusions. In addition, we studied the influence of the reaction mechanism in both the Tjon and Arenhövel nonrelativistic and relativistic models. The results in PWIA and distorted wave impulse approximation (DWIA) both with and without MEC and IC effects affect the cross section by less than $6 \%$ for missing momenta below $150 \mathrm{MeV} / c$ and less than 0.04 on $A_{\phi}$. Thus, the reaction mechanism appears sufficiently well behaved to allow a clear interpretation of our data.

Having established that the influence of FSI, MEC, and IC effects is small, we study the asymmetry $A_{\phi}$, which in PWIA is sensitive to the current operator only. The asymmetry $A_{\phi}$ is shown in Fig. 3. The shaded band indicates the systematic uncertainty, which is mainly

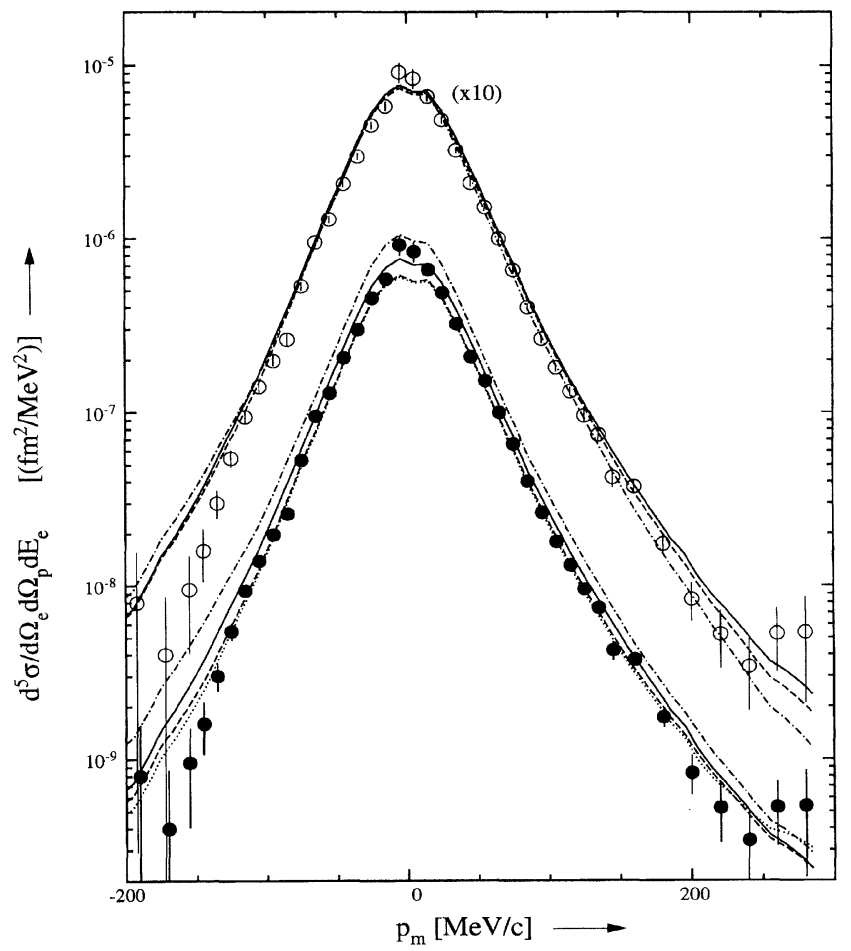

FIG. 2. The fivefold differential cross section for the reaction ${ }^{2} \mathrm{H}\left(e, e^{\prime} p\right) n$ as a function of the missing momentum at $Q^{2}=$ $1.2(\mathrm{GeV} / c)^{2}$. The upper (lower) curves represent the results of Tjon (Arenhövel). The dashed (dot-dashed) curves represent the relativistic (nonrelativistic) PWIA calculations, the solid curves represent $K \sigma_{c c 1} S_{\text {Paris }}$, and the dotted curve represents the relativistic calculation including FSI, MEC, and IC effects.

due to imperfect knowledge of the kinematics. Only a small asymmetry is observed at low missing momenta, since the component of the missing momentum parallel to the momentum transfer is not negligible. At low missing momenta the systematic uncertainty obscures the interpretation of the results. For $\left|\mathbf{p}_{m}\right|>70 \mathrm{MeV} / c$, the data clearly prefer the relativistic descriptions of $A_{\phi}$ over the nonrelativistic descriptions, but within the uncertainty no preference can be made between the relativistic models.

It is remarkable that the differences between the various models are sizable even in PWIA, reflecting the theoretical uncertainties in the treatment of the off-shell nuclear current operator. All nonrelativistic calculations yield an asymmetry that is approximately 4 times smaller than the relativistic calculations, indicating that an important ingredient is missing in the nonrelativistic reduction scheme when applied to the $L T$ interference strength. Note that $\sigma_{c c 1}$ of de Forest also yields a satisfactory description.

In summary, the present ${ }^{2} \mathrm{H}\left(e, e^{\prime} p\right) n$ experiment significantly extends the range of $Q^{2}$ compared to previous experiments. It is found that the $L T$ interference structure function is sensitive to the description of the nuclear current operator, and especially to relativistic ingredients 


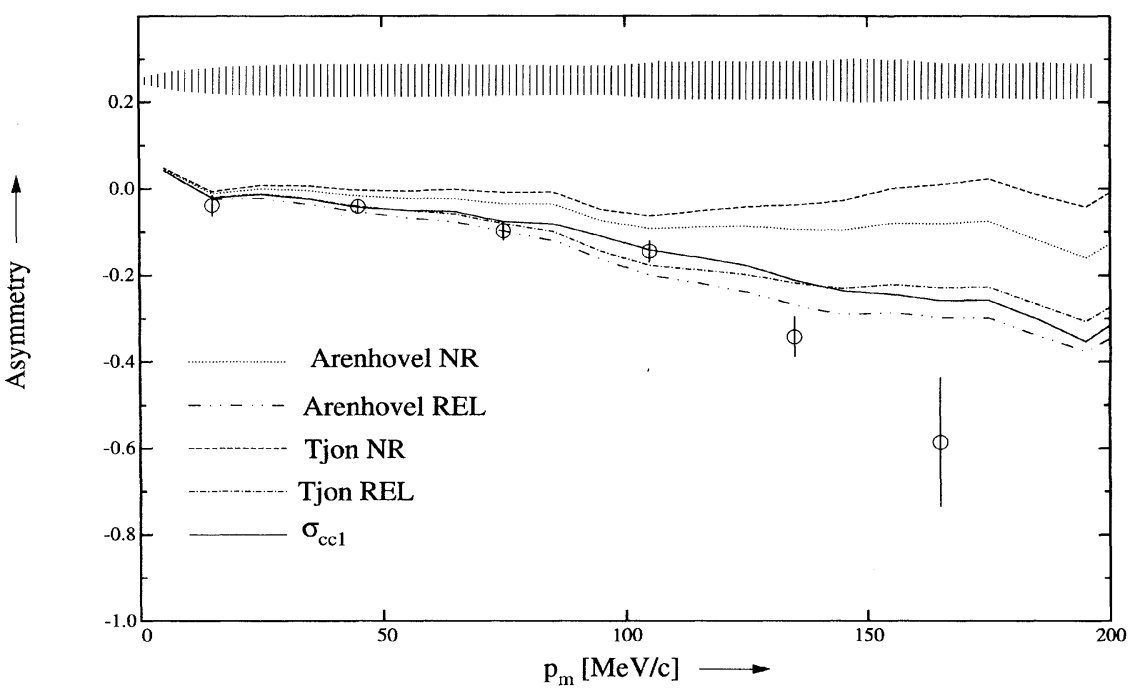

FIG. 3. The asymmetry $A_{\phi}$ for the reaction ${ }^{2} \mathrm{H}\left(e, e^{\prime} p\right) n$ as a function of the missing momentum at $Q^{2}=1.2(\mathrm{GeV} / c)^{2}$ for various nonrelativistic (upper set of curves labeled NR) and relativistic (lower set of curves labeled REL) models.

of the theory. The relativistic models of Refs. [12] and [14] give a satisfactory description of the asymmetry, whereas the nonrelativistic descriptions fail to do so. Both the $Q^{2}$ dependence of the cross section and the asymmetry can be described at the level of $10 \%$ in the PWIA approximation, using the $\sigma_{c c 1}$ off-shell prescription of de Forest and the Paris spectral function for deuterium.

We are grateful to Professor H. Arenhövel and Professor J. Tjon for performing calculations on a dense grid of kinematics, covering our experimental acceptance. This work was supported in part by the Department of Energy under Contracts No. W-31-109-ENG-38 (Argonne), No. DE-FG02-86ER40269 (Colorado), No. W-2705Eng-48 (LLNL), No. DE-AC02-76ER03069 (MIT), No. DE-AC03-76SF00515 (SLAC), No. DE-FG0388ER40439 (Stanford), and by the National Science Foundation under Grants No. PHY-9014406 and No. PHY-9114958 (American), No. PHY-9115574 (Caltech), No. PHY-9101404 (CSLA), No. PHY-9208119 (RPI), and No. PHY-9316221 (Wisconsin). R. G. M. acknowledges the support of a Presidential Young Investigator Award from NSF. B.W.F. acknowledges the support of a Sloan Foundation Fellowship.

*Present address: University of Maryland, College Park, MD 20742.

Present address: University of Colorado, Boulder, CO 80309.

${ }^{\ddagger}$ Present address: University of Michigan, Ann Arbor, MI 48109.

${ }^{\S}$ Present address: CEBAF, Newport News, VA 23606.

Present address: University of Illinois, Urbana, IL 61801.

TPresent address: Argonne National Laboratory, Argonne, IL 60439.

** Present address: Virginia Union University, Richmond, VA 23220.
${ }^{\dagger \dagger}$ Present address: Old Dominion University, Norfolk, VA 23529.

㧊Present address: University of Pennsylvania, Philadelphia, PA 19106.

${ }^{\S}$ Present address: Caltech, Pasadena, CA 91125.

"IIIPresent address: SLAC, Stanford, CA 94305.

III Present address: Kent State University, Kent, OH 44242.

[1] N. C. R. Makins et al., Phys. Rev. Lett. 72, 1986 (1994).

[2] L. Ya. Glozman et al., Phys. Lett. B 252, 23 (1990); P. Hoodbhoy and R. L. Jaffe, Phys. Rev. D 35, 113 (1987); A.E. L. Dieperink and P. J. Mulders, Nucl. Phys. A489, 627 (1988).

[3] S. Frullani and J. Mougey, Advances in Nuclear Physics (Plenum Press, New York, 1984), Vol. 14.

[4] M. van der Schaar et al., Phys. Rev. Lett. 68, 776 (1992).

[5] M. van der Schaar et al., Phys. Rev. Lett. 66, 2855 (1991).

[6] NPAS Users Guide, SLAC Report No. 269, 1984 (unpublished).

[7] N.C.R. Makins, Ph. D. thesis, Massachusetts Institute of Technology, Cambridge, 1994 (unpublished); T.G. O'Neill, Ph.D. thesis, California Institute of Technology, Pasadena, 1994 (unpublished).

[8] D. Wasson et al. (to be published).

[9] L. W. Mo and Y. S. Tsai, Rev. Mod. Phys. 41, 205 (1969); Y.S. Tsai, Rev. Mod. Phys. 46, 815 (1974).

[10] Here $p_{m}$ is assigned a positive (negative) sign if $\mathbf{p}^{\prime}$ is a larger (smaller) angle than $\mathbf{q}$ with respect to the beam in the scattering plane.

[11] T. de Forest, Jr., Nucl. Phys. A392, 232 (1983).

[12] J. A. Tjon, Few-Body Systems Suppl. 5, 17 (1992); E. Hummel and J. A. Tjon, Phys. Rev. C 42, 423 (1990); E. Hummel, Ph.D. thesis, Rijks Universiteit Utrecht, 1991 (unpublished).

[13] L. L. Foldy and S. A. Wouthuysen, Phys. Rev. 78, 29 (1950).

[14] G. Beck and H. Arenhövel, Few-Body Systems 13, 165 (1992). 